\title{
Intersektionale Perspektive(n) auf Disability, Gender und Madness Versuche einer Annäherung
}

\section{Zusammenfassung}

Ist die Utopie einer gemeinsamen sozialen Praxis, die ohne Kategorisierungen auskommt, möglich? Und wenn ja, wie? Dieser Artikel möchte unter Bezug auf die Disability Studies Tendenzen aufzeigen, wie wir uns an neue Formen des Denkens und Handelns herantasten können, die Menschen nicht länger aufgrund bestimmter Zugehörigkeiten definieren und hierarchisieren, sondern Räume eröffnen für Vielheiten und Nicht-Identitäten. Die im Folgenden dargelegten Gedanken sind Ergebnisse einer 2018 durchgeführten Diskursanalyse zu den intersektionalen Verwobenheiten von Nicht/Behinderung (Dis/ability), Geschlecht (Gender) und psychischer Gesundheit/ Krankheit (Madness). Herausgearbeitet wird, dass die hegemoniale Herstellung von Disability, Gender und Madness eng miteinander verschränkt geschieht und dass in deren Herstellung gleiche VerAnderungs-Narrative existieren und wirken. Weiter zeigt dieser Artikel diachrone Entwicklungen und Strategien des Dominanzdiskurses auf, die soziale Differenzen und Subjekte mit unterschiedlichen gesellschaftlichen Positionierungen etablieren und so minorisierte Andere erst generieren; bezieht aber ebenso die Antworten des Gegendiskurses auf diese Zustände mit ein.

\section{Approaching Intersectional Perspective(s) on Disability, Gender and Madness}

\begin{abstract}
English
Is the utopia of a common social practice without categorizations possible? And if so, how? Against the background of Disability Studies, this article shows how we can approach new forms of thinking and acting that no longer define and hierarchize people, but open spaces for multiplicities and non-identities. The ideas presented result from of a 2018 discourse analysis on the intersection of dis/ability, gender, and madness. It shows that the hegemonic production of disability, gender, and madness are closely intertwined and that similar narratives exist and operate in their production. Furthermore, the article illustrates how the dominant discourse establishes social differences and unequal subject positions and thus generates minoritized others in the first place.
\end{abstract}

Keywords: intersectionality, disability, gender, madness 


\section{Einleitung}

Eine intersektionale Betrachtung von Disability, Gender und Madness bietet einen Einblick in die Geschichte und die (Aus-)Wirkungen gesellschaftlicher Machtverhältnisse. Alle drei Begriffe werden jeweils als durch und in diesen diskursiv hergestellt betrachtet, weshalb sie als Analyseinstrumente für vorherrschende Praktiken der Diskriminierung und Exklusion dienen. ${ }^{1}$ Intersektionalität wird hier vor allem als methodischer Zugang ${ }^{2}$ verstanden der verdeutlicht, wie Kategorien und Zugehörigkeiten überhaupt erst entstehen konnten und welche Bedeutungen damit verknüpft sind (vgl. Gibson, 2015, S. 322). Dabei werden historische, themenbezogene und rhetorische Gemeinsamkeiten von Madness, Gender und Disability aufgezeigt und in einer Art Genealogie ihre Verwobenheiten nachgezeichnet. Die Darstellung basiert auf einer Diskursanalyse mit dem Titel „Remedy of Sorts? - herrschaftskritische Perspektiven der Antipsychiatrie, Queer Theory und Crip Theory", welche mit Hilfe des diskurshistorischen Ansatzes der Wiener Schule (Wodak \& Meyer, 2009) die intersektionale Verwobenheit von Disability, Gender und Madness untersucht. ${ }^{3}$ Die am Ende skizzierten Lösungsansätze zeigen Wege auf, wie sich an eine non-kategoriale Praxis herangetastet werden kann.

\section{Historische Intersektionalität, gemeinsames Erbe}

Das 19. Jahrhundert kann als der Beginn des Diskurses um Disability, Gender und Madness ${ }^{4}$ in dem Sinne bezeichnet werden, als dass sich mit dem Aufkommen von Wissenschaften wie der Psychologie oder der Sozialmedizin die Forschung an und zu Menschen, die als körperlich oder geistig abweichend galten, intensivierte (vgl. Zaviršek, 2000, S. 177ff). Die (Lebens-)Realitäten um Behinderung, Geschlecht und Psychiatrisierung wurden stärker in die öffentliche Sphäre gerückt (vgl. Zaviršek, 2000, S. 170f.) und so zum Gegenstand von politischen wie auch sozialmedizinischen Diskussion und Interessen (vgl. Berger \& Michel, 1997, S. 926).

Das Zusammenspiel von Disability, Gender und Madness lässt sich exemplarisch an der Hysterie, einer der zentralen Diagnosen der damaligen Zeit, verdeutlichen: Sie galt als Krankheit, die ausschließlich Frauen betraf bzw. als der Weiblichkeit immanent angesehen wurde: „The animal within them is desirous of procreating children, and when it remains unfruitful long beyond its proper time, gets discontented and angry, and [...] drives them to extremity, causing all varieties of disease" (Zaviršek, 2000, S. 178). Der Uterus wird als ein in der Frau wütendes Tier betrachtet, das, wenn es keine Nachkommen gebärt, die Frau krankt macht. Derartige Erklärungsmodelle waren wissenschaftlich anerkannt und dienten dazu, Hysterie, aber auch andere Abweichungen zur sexuellen und zweigeschlechtlichen Norm als psychische Störung, ,psychopathia sexualis', zu klassifizieren (vgl. Zaviršek, 2000). Die meisten Diagnosen unterlagen der Vorstellung von einer weiblichen Pathologie (vgl. Zaviršek, 2000, S. 171), die zusätzlich mit Attributen wie Sucht, Intelligenzminderung und Epilepsie (vgl. Zaviršek, 2000, S. 172ff) einherging, wodurch der intersektionale Bezug zu Disability deutlich wird. Die sich hier vollziehende VerAnderung von Geschlecht, psychischer Gesundheit/Krankheit und Behinderung verdeutlicht, wie stark die Prozesse der Minorisierung miteinander verknüpft sind. ${ }^{5}$ So wurde die Geschlechterdichotomie und -ungleichheit aufrechterhalten und gleichzeitig Frauen, aber auch behinderte und psychiatrisierte Menschen als abweichend, krank und bedrohlich definiert. Die Folge war nicht selten der Ausschluss aus der Gesellschaft (vgl. Block, 2000, S. 241).

Auffassungen wie jene legitimierten die Anfänge der psychiatrischen Institutionalisierung (vgl. Block, 2000, S. 241) von behinderten Menschen und jenen mit psychiatrischer Diagnose (vgl. Berger \& Michel, 1997, S. 930), Schwarzen und People of Colour (vgl. Gill \& Erevelles, 2017, S. 129), sowie von Armut betroffenen Witwen und unverheirateten Frauen (vgl. Zaviršek, 2000, S. 170f) zur Jahrhundertwende zum 20. Jahrhundert. Ziel war es zu verhindern, dass sich diese Personen fortpflanzten (vgl. Block, 2000, S. 241) und zu einer Bedrohung für die gesellschaftliche Ordnung würden (vgl. Block, 2000, S. 244).

Diese Befürchtungen mündeten zu Beginn des 20. Jahrhunderts in die Eugenik und „Euthanasie“ (vgl. Gibson, 2015, S. 319), die systematische Tötung sogenannten, lebensunwerten Lebens' (vgl. Berger \& Michel, 1997, S. 930). Auch die Zwangssterilisation, die nicht ausschließlich aber insbesondere an behinderten Frauen und 
jenen mit psychiatrischer Diagnose durchgeführt wurde (vgl. Block, 2000, S. 245), diente diesem Zweck. Jene Zeit verdeutlicht in extremer Form das Zusammenspiel intersektionaler Diskriminierung. Betrachten wir die Ausführungen im Sinne einer intersektionalen Perspektive, so wird deutlich, dass mit der Zwangssterilisation, und zu Teilen auch der "Euthanasie", genderspezifische bzw. misogyne diskriminierende Praktiken hervorgebracht und darüber hinaus Formen von psychischer Abweichung in einen Kausalzusammenhang mit Madness, nicht-binärem Gender, nicht-heterosexuellem Begehren, Disability, Race und Class gebracht wurden (vgl. Gibson, 2015, S. 319). ${ }^{6}$

Doch auch nach dem Ende des Zweiten Weltkriegs setzte sich das Interesse der Er- und Beforschung von Menschen, die als abweichend galten, fort. Auch sind medizinische Zwangsmaßnahmen, die ideologisch auf jener Zeit aufbauen, z.B. die Sterilisation von behinderten Frauen, keineswegs aus der Welt, bedienen sich nur einer anderen Legitimationsgrundlage, und zwar dem "Schutz des Individuums“ vor den Belastungen einer Schwangerschaft (Berger \& Michel, 1997, S. 931). Zweifelsohne spielt dabei auch die Befürchtung vor ökonomischen Belastungen für die Gesellschaft eine zentrale Rolle (vgl. Block, 2000, S. 246).

Die Nachkriegszeit bis in die 1980er Jahre ist stark vom Aufkommen der sozialen Bewegungen (vgl. Lewis, 2016, S. 112), wie der Frauen*Bewegung, der Behindertenbewegung, der Antipsychiatriebewegung und der Gay Pride Bewegung geprägt. Diese lösten eine sozialpolitische Wende aus und konnten Verbesserungen für Betroffene im Hinblick auf Stigmatisierung und Diskriminierung erwirken. Auch trugen sie zu einer Akademisierung der kritischen Wissenschaften, wie den Gender und Disability Studies und später auch den Mad Studies bei (vgl. Lewis, 2016, S. 98ff). Neben diesen Fortschritten zeigt sich aber ein Fortbestehen von Diagnosen des 19. Jahrhunderts in Manualen wie der ICD (International Statistical Classification of Diseases and Related Health Problems) (vgl. Krämer \& Tausch, 2015, S. 17) und dem DSM (Diagnostic and Statistical Manual of Mental Disorders) (vgl. Lukes, 2016, S. 335). In letzterem wurde der Begriff der ,Paraphilie' aus dem 19. Jahrhundert ab 1980 als Oberkategorie für Formen, sexueller Abweichung' wiedereingeführt und ist auch in der aktuellen Ausgabe vertreten (vgl. Lukes, 2016). Entwicklungen wie diese verdeutlichen Widersprüche und Ambivalenzen in Bezug auf die Geschichte von Disability, Gender und Madness. Denn aktivistische Bemühungen haben wesentlich zu gesellschaftlichen Veränderungen beigetragen und haben bewirkt, dass Menschen, die als abweichend galten, eine höhere Sichtbarkeit und im gleichen Zuge mehr Rechte erhalten. Gleichzeitig aber bleibt die Hoheit über (Be-)Deutungen weiterhin bei der Medizin und deren Klassifikationssystemen, die sich nach wie vor wirkmächtig in gesellschaftliche Diskurse einflechten.

Bereits seit den späten 1960er und frühen 1970er Jahren gehört innerhalb der aktivistischen Bewegungen und der Theoriebildung um Disability, Gender und Madness das Reclaiming zu einer zentralen Form des Widerstands gegen hegemoniale Zuschreibungspraxen (vgl. Lukes, 2016, S. 327). Begriffe wie ,queer', ,crip' und ,mad' - allesamt Termini, die eine Abweichung zur Norm und damit eine Herabwürdigung implizieren eigneten sich Betroffene wieder an, um sie als Instrumente der Abwertung dem Dominanzdiskurs zu entziehen. Ebenfalls gewinnt die intersektionale Theoriebildung (vgl. Guzmán \& Platero, 2014, S. 365) mehr und mehr an Bedeutung.

Medizinisch-psychiatrische Maßnahmen und Zuschreibungspraxen haben sich in den letzten sechzig Jahren stark gewandelt. Sie sind im Vergleich zum 19. Jahrhundert und insbesondere der Zeit der „Euthanasie“Morde im Nationalsozialismus anders gewaltvoll im Sinne von weniger direkten und offenkundigen Gewaltakten (Gibson, 2015, S. 321). Die grundlegende Unterscheidung normal/abweichend besteht indes weiter fort und bestimmt über den gesellschaftlichen Ein- oder Ausschluss: „The mutually constitutive definition of who is , fit' and who is ,unfit' continues to resonate in contemporary operations of difference. The legacy of eugenics persists in dominant [...] discourses about queerness, disability and social desirability" (Gibson, 2015, S. 322). 


\section{Interdiskursive Intersektionalität, gemeinsame Themen}

Die nun folgende Darstellung zur interdiskursiven Intersektionalität verdeutlicht Verflechtungen von Disability, Gender und Madness anhand von gemeinsamen thematischen Strängen. Dazu habe ich zwei eng miteinander in Zusammenhang stehende Themen, und zwar Körper und Sexualität, ausgewählt. Sie zeigen auf, welches gesellschaftliche Wissen im Kontext einer Überlappung (vgl. Guzmán \& Platero, 2014, S. 365) von Disability, Gender und Madness transportiert wird.

In den Disability, Gender und Mad Studies dominiert ein Verständnis von Körper als kultureller und sozialer Konstruktion; jegliche Machtverhältnisse und -strategien werden als in ihn eingeschrieben verstanden und Körper entsprechend mit Bedeutungen belegt (vgl. z.B. Fritsch, 2013, S. 6). Sexualität als Teil von Körperlichkeit ist folglich auch eine soziale, von gesellschaftlichen Bedingungen geformte Praxis. Die Sexualität von minorisierten Menschen wird im Dominanzdiskurs als entweder nicht existent, unmöglich oder aber als übermäßig und unverhältnismäßig beschrieben. Die Zuschreibung „asexuell“ (z.B. Guzmán \& Platero, 2014, S. 378) findet sich vielfach in Bezug auf behinderte Menschen, (lesbische) Frauen, Trans*Menschen und vor allem behinderte Frauen. Sexualität zu leben sei für behinderte Frauen gar nicht möglich, da sie nicht begehrenswert seien und körperliche Einschränkung sexuelle Aktivität ausschließe (vgl. Guzmán \& Platero, 2014, S. 374ff). Das andere Extrem unterstreichen Begriffe wie „sexual monsters" (Block, 2000, S. 251) und allen voran „pervers“7 (Baril \& Trevenen, 2014, S. 407) zur Beschreibung der Sexualität von behinderten Menschen und Trans*Menschen. Es dominieren Bilder der Ent- oder Hypersexualisierung.

Um diese Argumentation zu konkretisieren, möchte ich im Folgenden beispielhaft eine spezifische Intersektion von Disability, Gender und Madness betrachten: Beim Thema Amelotatismus ${ }^{8}$, dem sexuellen Begehren nichtbehinderter Männer von Frauen mit amputierten Gliedmaßen, stehen zunächst deutlich Disability und Gender im Vordergrund, und zwar im gegendiskursiven Sinne, denn körperliche Differenz, die sonst eher pathologisiert wird, gilt hier als sexuell begehrenswert (vgl. Solvang, 2007, S. 52). Bei genauerer Betrachtung aber wird eine heteronormative Objektivierung erkennbar, und auch das Potential, Disability im Sinne von Attraktivität umzudeuten, ist begrenzt, da die körperliche Abweichung das entscheidende Kriterium für das Begehren des Devotees ist: „If the male gaze makes the normative female a sexual spectacle, then the stare sculpts the disabled subject into a grotesque spectacle. The stare is the gaze intensified, framing her body as an icon of deviance" (Solvang, 2007, S. 54). Der Bezug zu Madness stellt sich bei Amelotatismus dadurch her, dass diese Form des Begehrens unter der Diagnose ,Akrotomphilie' als eine psychische Störung angesehen wird (vgl. Solvang, 2007, S. 52). Eine ähnliche Überlappung von Zuschreibungen zeigt sich bei ,Transability', dem Wunsch nach behinderter Körperlichkeit (vgl. Baril \& Trevenen, 2014). Ebenfalls psychiatrisch klassifiziert, wird sie als ,Körperintegritätsidentitätsstörung' oder „Apotemnophilie' bezeichnet (vgl. Baril \& Trevenen, 2014, S. 398). Die Diagnosen ,Apotemnophilie’ und ,Akrotomphilie' verweisen auf die in der Medizin verankerte, zutiefst ableistische Annahme, dass Disability (sexuell) zu begehren bedeutet, psychisch krank zu sein (vgl. Baril \& Trevenen, 2014, S. 406; Solvang, 2007, S. 52).

\section{Rhetorische Intersektionalität, gemeinsame (Be-)Deutungen}

Neben den Betrachtungen zu historischen und thematischen Verflechtungen kommt auch der Rhetorik, die im Kontext von Disability, Gender und Madness verwendet wird, eine entscheidende Rolle zu. Sprachliche Bilder sind machtvolle Instrumente. Auch sie stehen in einem dialektischen Verhältnis zu vorherrschenden gesellschaftlichen Praxen und haben damit erheblichen Einfluss darauf, wie über die bezeichneten Menschen gedacht wird. Folgende Darstellungen von Disability, Gender und Madness werden im Mehrheitsdiskurs (re-)produziert: Eine sehr gängige Darstellung von Menschen, die als abweichend klassifiziert werden, ist die der Gefahr bzw. der Gefährdung. Frauen, queere und psychiatrieerfahrene Menschen gelten als exzessiv (z.B. Baril \& Trevenen, 2014, S. 407) und aggressiv (vgl. Wolframe, 2013). Psychiatrieerfahrenen wird „unpredictable violence“ (Wolframe, 2013) zugeschrieben, autistischen Menschen eine „destructive force“ (Bumiller, 2008, S. 970). Weitere Bilder der Entmenschlichung sind Tiervergleiche: „Piggy” (Block, 2000, S. 
248), "she just cawed and chirped like a bird“ (Frauen mit Behinderung), ",his hairs were grown like eagles' feathers, and his nails like bird claws" (Madness) (Donaldson, 2002, S. 109). Sogar die Figur des Dämons (vgl. Block, 2000, S. 248) als auch die des Monsters finden Erwähnung (z.B. vgl. Lewis, 2016, S. 108). Diese Darstellungen vermitteln vor allem ein Gefühl der Bedrohung. Die drastischen Herabwürdigungen dienten dem Ziel, den Ausschluss jener Personengruppen zu rechtfertigen und boten damit u.a. der Eugenik eine Legitimationsgrundlage (vgl. Berger \& Michel, 1997, S. 926). Auch finden sich Vergleiche zu Maschinen: "remarkably machine-like rather than human body" (Bumiller, 2008, S. 970), und, insbesondere im Kontext von Madness, allegorische Figuren wie "prophets" oder "mad geniuses" (Wolframe, 2013). Derartige Überstilisierungen, auch wenn unter Umständen positiv deutbar, verunmenschlichen gleichermaßen.

Auch Ortsmetaphern finden in diesem Diskurs Anwendung. In Bezug auf Disability, Gender und Madness markieren sie sowohl Exklusion als auch Abweichung. So ist in englischen Quellen die Rede von "far-offplaces” (Miller, 2017, S. 6), „alien-territory”, „no-man's land” (Miller, 2017, S. 10) (Madness), und "regions forsaken by thought” (Lewis, 2016, S. 111) (allgemein). „A little bit off“" (Lewis, 2016, S. 83) und "on the fringes" (Wolframe, 2013) (Madness) beschreibt eine marginalisierte Existenz. In Bezug auf Disability bezeichnen Orte auch den Körper, „Alien-land“ (Fritsch, 2013, S. 12), oder Ängste, „the social realm is viewed as a treacherous territory" (Bumiller, 2008, S. 980).

Jene Sprechakte der Ausgrenzung prägen den Dominanzdiskurs um Disability, Gender und Madness nach wie vor entscheidend. Insgesamt konnten die letzten Ausführungen zeigen, dass die VerAnderung von Menschen, die als abweichend gelten, in vielen gesellschaftlichen Strukturen tief verankert ist und trotz aller Veränderungen und Verbesserungen weiter fortwirkt und Benachteiligung und gesellschaftlichen Ausschluss zur Folge hat. Eine intersektionale Betrachtung dieser Exklusionsmechanismen konnte zeigen, dass diese Prozesse miteinander verwoben sind und immer gleichzeitig mehrere Strukturen betreffen, wodurch sie an Wirkmächtigkeit gewinnen.

\section{Intersektionale Perspektive, gemeinsame Zukunft}

Die folgenden Ausführungen sind Antworten des Gegendiskurses auf diese bestehenden Verhältnisse. Sie zeigen Tendenzen auf und bieten Ansatzpunkte für die Beantwortung der eingangs gestellten Fragen, ob und wie eine non-kategoriale soziale Praxis möglich ist. Nachstehende Entwürfe des Handelns und Sprechens können dazu beitragen.

\section{Solidarität}

Insgesamt wird einem Aktivismus, der Machtverhältnisse und Zuschreibungspraxen als Ganzes in Frage stellt (Krämer \& Tausch, 2015, S. 8), ein großes Potential zugeschrieben. Ziel ist eine intersektional solidarische Bewegung, die über (Identitäts-)Grenzen hinweg Widerstand ermöglicht und nicht zu (Re-)Pathologisierung an anderer Stelle führt (vgl. Krämer \& Tausch, 2015, S. 2). Unter Solidarität sind auch konkrete Konzepte zu verstehen, die Betroffene stärker in Entscheidungsprozesse miteinbeziehen, z.B. Peer-Support (vgl. Joseph, 2013, S. 278) oder Trialog (vgl. Joseph, 2013, S. 280). Solidarität kann auch ein neuartiges Verständnis von Selbstbestimmung bewirken, eines, das Menschen selbst überlässt, „wann sie den gängigen Interpretationen von und als ,Krankheiten' folgen wollen und wann sie ihre Pathologisierung ablehnen und dekonstruieren möchten“ (Boger, 2015, S. 288).

\section{Erfahrungen teilen}

Erfahrungen zu teilen, ermöglicht für Betroffene Sichtbarkeit und Handlungsfähigkeit; auf Seiten der Zuhörer_innen führt sie zu Reflexion (vgl. Nabbali, 2015, S. 2). Das Konzept des ,storytelling', bei dem minorisierte Menschen ihre eigene (Lebens-)Geschichte erzählen, kann vorherrschende normative Narrative 
verändern und Räume eröffnen für die Komplexität von Identität(en) (vgl. Nabbali, 2015). Keine Lebensgeschichte und -erfahrung lässt sich mit starren Kategorien beschreiben.

Auch für die Wissenschaft sind Erfahrungen von Belang, was im Konzept des, Haunting' (vgl. Gill \& Erevelles, 2017) deutlich wird. Zunächst eilt dem Begriff und der damit in Verbindung stehenden Figur ,Ghost' etwas Unheimliches voraus. Der Spuk und der Geist sind gleichzeitig da und nicht da (vgl. Gill \& Erevelles, 2017, S. 124); und genau darum geht es. Haunting kann als ein analytisches Werkzeug dienen, die Geschichte(n) derer, die unsichtbar (gemacht worden) sind, aber dennoch - oder gerade deshalb - Unbehagen auslösen, aufzuspüren, aufzudecken und den vorherrschenden Diskurs durch deren neu erlangte Sichtbarkeit zu erschüttern: "It is through haunting that the dead are never content to stay buried" (Hong; zit. n. Gill \& Erevelles, 2017, S. 135). Dadurch kommt der Figur des ,Ghost' eine neue Bedeutung zu, und zwar die einer "social figure who holds in its elusive form both the absence and presence of its history" (Hong; zit. n. Gill \& Erevelles, 2017, S. 134). In ihr vereinen sich sowohl die Unsichtbarkeit als auch die Sichtbarkeit ihrer Geschichte, weil sie vermeintlich plötzlich in Erscheinung tritt und dies Fragen nach ihrer Vergangenheit aufwirft. Diese Perspektive auf die Geschichte unsichtbarer/minorisierter Gruppen anzuwenden, bedeutet sich mit den gesellschaftlichen Machtverhältnissen auseinanderzusetzen, die deren Unsichtbarkeit hervorgerufen haben.

\section{Neuform(ul)ierungen}

Dieser Begriff beinhaltet Versuche der Umdeutung. Sie finden sich in Neologismen, Konzepten und bei der Betrachtung von allegorischen Figuren, so z.B. des ,Cyborg', "a radical border-crosser, blurring the boundaries between human and animal, machine and organism, physical and non-physical" (Kafer, 2013, S. 103). Das, worauf der Cyborg verweist, ist das gleichzeitige Auftreten von widersprüchlichen Attributen; er ist ein hybrides Wesen, das sich jeglicher äußeren Bestimmung entzieht (vgl. Kafer, 2013). Bezogen auf Disability, Gender und Madness liefert diese analytische Figur die Erkenntnis, dass jegliche Form(en) der Identität gleichzeitig viele andere miteinschließen und sie sich in einem stetigen Veränderungsprozess befinden (vgl. Kafer, 2013).

Was hier mitschwingt, ist das Thema Zeitlichkeit, das auch Teil des von Jack Halberstam entwickelten Konzepts ,Queer Time' ist (Dalke \& Mullaney, 2014). Dieses bezeichnet das Unterwandern normativer Lebensentwürfe, wie z.B. das der heterosexuellen Kleinfamilie, die in den meisten Fällen queere (und auch behinderte und psychiatrisierte) Menschen ausschließen. Menschen, denen diese Modelle verwehrt bleiben, arrangieren sich fernab jeglicher ,Chrononormativität', wodurch „imaginative life schedules” (Samuels; zit. n. Dalke \& Mullaney, 2014), sinngemäß selbst erdachte Lebensentwürfe, entstehen und verwirklicht werden können.

Im Kontext von Disability gibt es analog zur Queer Time das Konzept ,Crip Time' (Dalke \& Mullaney, 2014), das sich bewusste Widersetzen gegenüber der gesellschaftlichen Vorstellung, dass Behinderung geheilt werden müsse. Der (Lebens-)Zeit von behinderten Menschen wird hier eine neue Bedeutung zugeschrieben, nämlich die, dass der Mensch diese nicht nutzt, um, wie es oft erwartet wird, sich der Heilung oder Schonung zu widmen, sondern eben der Behinderung „ihre“ Zeit zu geben, und das kann auch bedeuten, nichts zu tun: "Some time that [...] is just plain wasted... sometime we are just ,'doing time' - in depression, in illness, in times when there is nothing really beyond surviving to do" (Dalke \& Mullaney, 2014). Um zu verdeutlichen, was gemeint ist, lässt sich der akademische Kontext beispielhaft heranziehen. Dieser schließt insbesondere behinderte und psychiatrisierte Menschen aufgrund strikter zeitlicher Vorgaben aus und sieht nicht vor, dass Menschen aufgrund von Einschränkungen mehr Zeit benötigen (vgl. Dalke \& Mullaney, 2014). Crip Time würde in diesem Zusammenhang bedeuten, über die Möglichkeit Nachteilsausgleiche einzufordern und Abgabefristen zu verlängern, hinauszugehen, und "unproduktive“ und "leere" Zeiten (vgl. Dalke \& Mullaney, 2014) genauso in Arbeitsprozesse miteinfließen zu lassen. So sollen die Lücken, die sich im System Universität auftun, für eine Umdeutung genutzt werden: "to discover gaps in which normative time is ruptured, suspended [...] to make the academy more welcoming of difference, and more able to acknowledge multiple 
modes of failure and achievement" (Dalke \& Mullaney, 2014). An die Stelle der Dichotomie krank/gesund tritt Diversität (vgl. Dalke \& Mullaney, 2014).

Auch auf der rein sprachlichen Ebene können Umdeutungen vollzogen werden. Neologismen werden gebraucht, um subversive Strategien zu benennen, „entzweigendern“ (Hornscheidt \& Lüthi, 2018, S. 18), "Rück_Aneignung" (Krämer \& Tausch, 2015, S. 10) oder "queering, cripping, maddening“" (Dalke \& Mullaney, 2014) und um neue Benennungspraxen aufzuzeigen: "mindfreedom", "transminded" und "dis-labeled" (Dalke \& Mullaney, 2014). Um den pathologisierenden Zuschreibungen des Mehrheitsdiskurses entgegenzuwirken, werden neutrale Konnotationen bezüglich Disability, Gender und Madness vorgeschlagen: „Symptoms of life” (Dalke \& Mullaney, 2014), „, functional diversity” (Guzmán \& Platero, 2014, S. 358) (Disability), ",gender variant/fluidity” (Saketopoulou, 2011, S. 193) (Gender), „psychiatric survivor” (Pilling, 2013, S. 3) (Madness). All diese verdeutlichen Vielfalt und Differenz als konstitutiv für Identität(en) und verweisen auf die Prozesshaftigkeit von Körpern und Leben.

Letzte Betrachtungen verdeutlichen, dass eine intersektionale Perspektive neben der Analyse historischer, thematischer und rhetorischer Verwobenheiten auch neue Wahrnehmungsformen ermöglicht, die im ersten Schritt zu einer Umkategorisierung und letztlich zu einer entkategorisierenden Wirklichkeit beitragen können. Es ist zwar kein leichtes und unmittelbar greifbares Unterfangen, eine Welt ohne Kategorien zu denken, geschweige denn zu schaffen; aber es sind eben solche Anfänge, die Schaffung von solidarischen Strukturen, die Beachtung bislang ungehörter (Lebens)Geschichten und die Bemühungen um neue Konzepte und Begriffe, die Utopien entstehen lassen, die weitergedacht werden und an denen sich Stück für Stück (Lebens-)Welten materialisieren können.

\section{Anmerkungen}

1 Disability und Madness werden in diesem Text als zwei unterschiedliche Positionsbestimmungen betrachtet, um aufzuzeigen, wie sie sich spezifisch herausbildeten und mit welchen jeweiligen Bedeutungen sie belegt sind.

${ }^{2}$ Die hier kurz umrissene methodische Anwendung einer intersektionalen Betrachtungsweise zur Analyse eines Diskurses stellt ein weiteres Ergebnis der Diskursanalyse dar. Das Textmaterial des Korpus mit der Fülle an diskursiven Verschränkungen bot die Möglichkeit, Intersektionalität als Methode neu zu erproben.

${ }^{3}$ Die Diskursanalyse wurde im Rahmen meiner Masterarbeit zum Studiengang „Praxisforschung in der Sozialen Arbeit und Pädagogik" an der Alice-Salomon-Hochschule Berlin durchgeführt. Innerhalb eines mehrstufigen Erhebungsverfahrens wurde aus einem anfänglichen Korpus von ca. 250 Fachartikeln aus der Soziologie, den Sozialwissenschaften und der Psychologie ein finaler Analysekorpus von 45 Artikeln erstellt. Die Artikel wurden über Fachdatenbanken wie EBSCOhost und DZI SoLit sowie das Zeitschriften-Archiv der Alice-Salomon-Hochschule gesucht. Kriterien für die Auswahl waren die Verwendung bestimmter Termini, die mit den jeweiligen Kategorien in Zusammenhang stehen und die ich im Vorfeld im Rahmen der theoretischen Fundierung der Arbeit herausgearbeitet hatte. Diese wurden mit den Booleschen Operatoren jeweils zueinander in Zusammenhang gesetzt. Die Wortfrequenzanalyse gab letztlich Aufschluss darüber, welche Artikel insbesondere das Thema fokussieren, die dann in die Arbeit miteinflossen.

${ }^{4}$ Die Begriffe Disability, Gender und Madness, wie sie in diesem Text verstanden werden, etablierten sich erst im Zusammenhang mit dem Aufkommen der jeweiligen Studies. Während die Gender und Disability Studies in den 1970er bzw. 1980er Jahren ihre Anfänge fanden, bilden sich die Mad Studies erst seit den letzten 10 Jahren als eigenes Forschungsfeld heraus.

${ }^{5}$ Ich wähle für diesen Text die Bezeichnungen minorisierte, behinderte und psychiatrisierte Menschen. Diese Schreibweise verdeutlicht die Konstruiertheit dieser Positionen. 
${ }^{6}$ In Bezug auf die Positionsbestimmungen Race und Class wirken die gleichen Machtstrategien.

${ }^{7}$ In diesem Diskurs wird der Begriff der Perversion auch im Sinne des Reclaiming als subversive Strategie behandelt (vor allem vgl. Lukes, 2016).

8 Dem Begriff Amelotatismus entspricht im Englischen "devotism", dieser Terminus wird in den hier herangezogenen Quellen verwendet. Für meine Ausführungen verwende ich den deutschen Begriff.

\section{Literatur}

Baril, A., \& Trevenen, K. (2014). Exploring ableism and cisnormativity in the conceptualization of identity and sexuality "disorders". Annual Review of Critical Psychology, 11, 389-416.

Berger, E., \& Michel, B. (1997). Zwangssterilisation bei geistiger Behinderung. Wiener Klinische Wochenschrift, 109(23), 925-931.

Block, P. (2000). Sexuality, fertility, and danger: Twentieth-century images of women with cognitive disabilities. Sexuality \& Disability, 18(2), 239-254. https://doi.org/10.1023/A:1005642226413

Boger, M. (2015). Das Trilemma der Depathologisierung. In C. Schmechel, F. Dion, K. Dudek, \& M. Roßmöller (Hrsg.), Gegendiagnose. Beiträge zur radikalen Kritik an Psychologie und Psychiatrie (S. 268288). Edition assemblage.

Bumiller, K. (2008). Quirky citizens: Autism, gender, and reimagining disability. Signs: Journal of Women in Culture and Society, 33(4), 967-991. https://doi.org/10.1086/528848

Dalke, A., \& Mullaney, C. (2014). On being transminded: Disabling achievement, enabling exchange. Disability Studies Quarterly, 34(2). https://doi.org/10.18061/dsq.v34i2.4247

Donaldson, E. (2002). The corpus of the madwoman: Toward a feminist disability studies theory of embodiment and mental illness. NWSA Journal, 14(3), 99-120.

Fritsch, K. (2013). On the negative possibility of suffering: Adorno, feminist philosophy, and the transfigured Crip to come. Disability Studies Quarterly, 33(4). https://doi.org/10.18061/dsq.v33i4.3869

Gibson, M. (2015). Intersecting deviance: Social work, difference and the legacy of eugenics. British Journal of Social Work, 4(5), 313-330. https://doi.org/10.1093/bjsw/bct131

Gill, M., \& Erevelles, N. (2017). The absent presence of Elsie Lacks: Hauntings at the intersection of race, class, gender, and disability. African American Review, 50(2), 123-137. https://doi.org/10.1353/afa.2017.0017

Guzmán, P., \& Platero, R. L. (2014). The critical intersections of disability and non-normative sexualities in Spain. Annual Review of Critical Psychology, 11, 357-387.

Hornscheidt, L., \& Lüthi, E. (2018). Die Macht sprachlicher Handlungen für Vorstellungen von Gender und Psychiatrie. Sozialpsychiatrische Informationen, 48(1), 15-19.

Joseph, A. (2013). Empowering alliances in pursuit of social justice: Social workers supporting psychiatricsurvivor movements. Journal of Progressive Human Services, 24(3), 265-288.

https://doi.org/10.1080/10428232.2010.540748 
Kafer, A. (2013). The Cyborg and the Crip. Critical encounters. In A. Kafer (Hrsg.), Feminist Queer Crip (S. 103-128). Indiana University Press.

Krämer, K., \& Tausch, S. (2015) De_Pathologisierung und_oder Re_Stigmatisierung? Eine Analyse von Wirkungsweisen hegemonialer Denkstrukturen in Gegendiskursen [Seminararbeit, HumboldtUniversität zu Berlin]. Digitale Bibliothek bidok - behinderung inklusion dokumentation. http://bidok.uibk.ac.at/library/kraemer-de_pathologisierung-.html

Lewis, A. (2016). "We are certain of our own insanity": Antipsychiatry and the Gay Liberation Movement, 1968-1980. Journal of the History of Sexuality, 25(1), 83-113. https://doi.org/10.7560/JHS25104

Lukes, H. (2016). Perversion, terminable and interminable: Foucault, Lacan, and DSM-5. Discourse: Journal for Theoretical Studies in Media and Culture, 38(3), 327-355. https://doi.org/10.13110/discourse.38.3.0327

Miller, G. (2017). Madness decolonized?: Madness as transnational identity in Gail Hornstein's Agnes's Jacket. Journal of Medical Humanities, 39(3), 1-21. https://doi.org/10.1007/s10912-017-9434-8

Nabbali, E. (2015). ID politics: The violence of modernity. Intersectionalities: A Global Journal of Social Work Analysis, Research, Polity, and Practice, 4(1), 1-14. https://journals.library.mun.ca/ojs/index.php/IJ/article/view/1518/1213

Pilling, M. (2013). Invisible identity in the workplace: Intersectional madness and processes of disclosure at work. Disability Studies Quarterly, 33(1). http://dx.doi.org/10.18061/dsq.v33i1.3424

Saketopoulou, A. (2011). Minding the gap: Intersections between gender, race, and class in work with gender variant children. Psychoanalytic Dialogues, 21(2), 192-209.

https://doi.org/10.1080/10481885.2011.562845

Solvang, P. (2007). The amputee body desired: Beauty destabilized? Disability re-valued? Sexuality and Disability, 25(2), 51-64. https://doi.org/10.1007/s11195-007-9036-x

Wodak, R., \& Meyer, M. (2009). Critical Discourse Analysis: History, agenda, theory and methodology. In R. Wodak, \& M. Meyer (Hrsg.), Methods of Critical Discourse Analysis (2. Auflage, S. 1-33). Sage.

Wolframe, P. (2013). The madwoman in the academy, or, revealing the invisible straightjacket: Theorizing and teaching saneism and sane privilege. Disability Studies Quarterly, 33(1). http://dx.doi.org/10.18061/dsq.v33i1.3425

Zaviršek, D. (2000). A historical overview of women's hysteria in Slovenia. European Journal of Women's Studies, 7(2), 169 - 188. https://doi.org/10.1177/135050680000700203

\section{Autor_in}

Anna-Catharina Heidrich, geb. 1983 in Berlin, ist studierte Heilpädagogin (B.A.) und Praxisforscherin (M.A.). Seit 2004 arbeitet sie in unterschiedlichen Bereichen der Behindertenhilfe, zuletzt in der antipsychiatrisch ausgerichteten Sozialen Arbeit. Seit Herbst 2020 ist sie als Beraterin in der Antidiskriminierungsberatung Alter, Behinderung, chronische Erkrankung der Landesvereinigung Selbsthilfe Berlin e.V. tätig. Als behinderte und queere Frau* engagiert sie sich vor allem in antisexistischen und antiableistischen Kontexten. All diese Erfahrungen haben ihre kritische Haltung gegenüber gesellschaftlichen Zuschreibungspraxen gefestigt und sie sieht sich als Vertreterin von und Kämpferin für Gegenentwürfe und Strategien, die ein Denken und Handeln fernab jeglicher Kategorisierungen bedeuten.

E-Mail: annaheidrich@gmx.de 